Bangladesh J. Plant Taxon. 13(2): 131-137, 2006 (December)

\title{
PLEUROCARPOUS MOSSES OF BANGLADESH : FAMILY ENTODONTACEAE
}

\author{
Hamida Khatun ${ }^{1}$ and Syed Hadiuzzaman \\ Department of Botany, University of Dhaka, Dhaka-1000, Bangladesh
}

Key words: Pleurocarpous mosses, Hypnobryales, Entodontaceae, Bangladesh

\begin{abstract}
Three species, namely, Erythrodontium julaceum, Pterigynandrum decolor and Entodon flavescens under family Entodontaceae are described with illustrations and a short note on each.
\end{abstract}

\section{Introduction}

Recent studies on the pleurocarpous mosses of Bangladesh (Khatun and Hadiuzzaman 1994, 1995, 2003, 2004a, 2004b, 2005a, 2005b, 2006) revealed that among different groups of pleurocarpous mosses, the members of the family Entodontaceae are not common. Tixier (1967) studied some bryophytes based upon some collections from Kaptai, Cox's Bazar and hills of Sitakund in Bangladesh. He reported a number of pleurocarpous mosses only in the form of checklist which included Erythrodontium julaceum from Entodontaceae.

Nonetheless, a recent study on pleurocarpous mosses of Bangladesh revealed that the family Entodontaceae of the order Hypnobryales is represented by three species under three genera and these are Erythrodontium julaceum, Pterigynandrum decolor and Entodon flavescens. In his monograph on mosses of eastern India and the adjacent regions, Gangulee described all the three species (Gangulee 1980), but did not give any information on their occurrence in Bangladesh. Furthermore, he mentioned Entodon flavescens as endemic to the Himalayas and Pterigynandrum decolor as an endemic species of eastern Himalayas.

In this paper three species, namely, Erythrodontium julaceum, Pterigynandrum decolor and Entodon flavescens, belonging to the family Entodontaceae have been described and illustrated along with their distribution. An artificial key to the genera and species, and distinguishing characters of each species are also given here.

\section{Family: Entodontaceae}

Medium-sized, slender, glossy plants in loose tuft. Main stem creeping, irregularly to pinnately or sub-pinnately branched, branches generally julaceous. Stem and branch leaves more or less similar, leaves crowded in many rows, usually appressed at least when dry, sometimes more or less complanate, mostly symmetric of various shape. Costa

${ }^{1}$ Corresponding author. 
none or very short, double and delicate. Leaves linear to oblong-linear, smooth, those at the basal angle quadrate and rectangular or transversely rectangular, sometimes in many oblique rows, alar very much differentiated.

\section{Key to the genera of Entodontaceae:}

1. Leaf cells smooth, alar cells forming a large conspicuous group extending obliquely about half way up the margins

Erythrodontium

- Cells smooth or papillose, alar not as above

2. Alar cells in many rows, quadrate, upper leaf cells short-rhomboid

Pterigynandrum

- $\quad$ Alar cells not in many rows, irregular in shape, upper leaf cells not shortrhomboid but elongated

Entodon

Genus: Erythrodontium Hamp. in Vid. Medd. Naturh.

For. Kjobenh. ser. 3, 2: 279 (1870)

Slender to moderately robust. Stem elongate, branch rigid, more or less julaceous. Leaves imbricate when dry, broadly oval or ovate-oblong, shortly apiculate from a strong decurrent base. Leaf cells narrowly elliptic, alar cells in oblique series, rounded-quadrate or transversely rectangular, forming a large conspicuous group extending obliquely about half length of leaf.

1. Erythrodontium julaceum (Schwaegr.) Par. in Index. Bryol.: 436 (1896)

(Plate 1)

Neckera julacea Hook. ex Schwaegr. in Sp. Musc. Suppl. 3(1): 245 (1828)

Pterogonium squarrosum Griff. in Cal. J. Nat. Hist. 3: 63 (1843)

Pterogonium squarrosulum Mont. in Lond. J. Bot. 4: 9 (1845)

Leptohymenium julaceum (Schwaegr.) Hamp. in Linnaea 20: 83 (1847)

Pterogonium julaceum (Schwaegr.) Hook. in C. Muell. Syn. 2: 101 (1850)

Neckera squarrulosa C . Muell in Syn., 2: 101 (1850)

Pterogonium squarrulosum Mont. in Syn.: 21(1856) ortho. err.

Stereodon juliformis Mitt. in Musci Ind. Or.: 92 (1859)

Platygyrium julaceum (Schwaegr.) Bosch \& Lac. in Bryol. Java. 2: 107, 217 (1864)

P. squarrosulum (Mont.) Jaeg. in Ber. S. Gall. Naturw.Ges. 1876-77: 277 (1878)

Entodon julaceus (Schwaegr.) C. Muell. in Linnaea 42: 435 (1879)

Erythrodontium juliforme (Mitt.) Par. in Index Bryol.: 436 (1896)

Erythrodontium squarrulosum ( Mont.) Par. in Index Bryol.: 437 (1896)

Plant rigid, glossy, golden-green, brown in old, slender to moderately robust. Stem elongate, prostrate, $5 \mathrm{~cm}$ or more long, branched, branches narrow, short, irregularly closely pinnate, julaceous, erect. Leaves dense, terete, imbricate, closely appressed to stem when dry, oval or ovate-oblong when moist, c. $1.04 \mathrm{~mm}$ long and $0.6 \mathrm{~mm}$ wide with suddenly narrowed short tip from a decurrent base, margin entire, nerveless. Leaf cells narrowly elliptic to linear c. $37.92 \times 13.2 \mu \mathrm{m}$ at tip, c. $57.7 \times 8.2 \mu \mathrm{m}$ at middle. Alar 
rounded-quadrate, transversely rectangular, c. $17.5 \times 8.7 \mu \mathrm{m}$ forming large conspicuous triangular patches of cells extending obliquely about half way up the margins (length of the leaf) render it easily distinguishable.

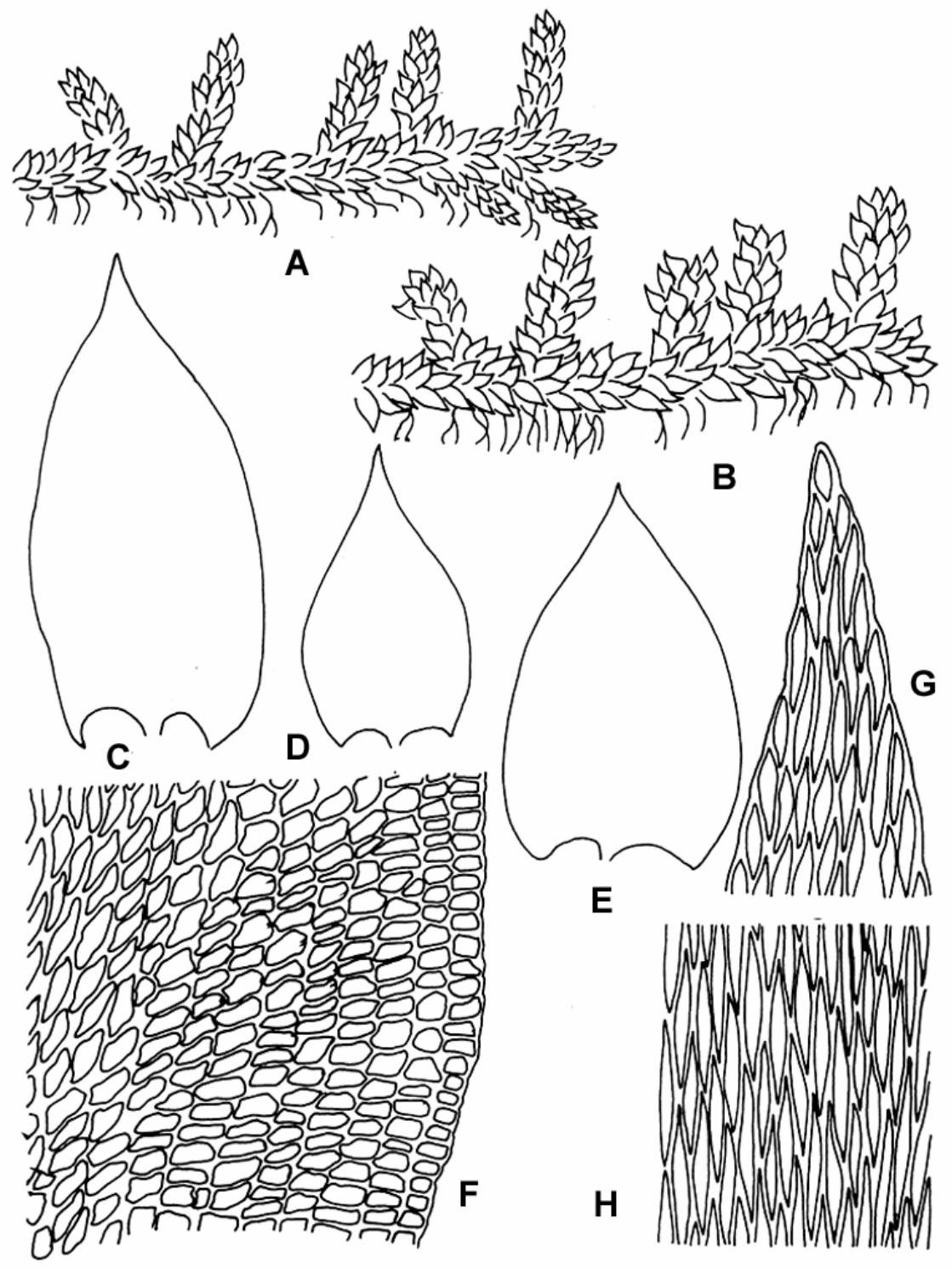

Plate 1. Erythrodontium julaceum (Schwaegr.) Par. A. Dry plant (×6.67), B. Wet plant (×6.67), C-E. Leaves $(\times 24)$, F. Basal laminal cells $(\times 200)$, G. Apical laminal cells $(\times 200)$, H. Middle laminal cells $(\times 200)$.

Specimens examined: This species is widely distributed all over the country. However, specimens were collected from the following districts: Barisal (656, 660, 768), 
Brahmanbaria (155), Bhola (728, 1336), Bogra (1295), Chittagong (760), Dhaka (757, 1004), Faridpur (514, 1441), Rangamati (693), Jamalpur (1292, 1323), Jessore (695, 1423), Khulna (1322), Kushtia (692), Madaripur (701), Moulvi Bazar (1287, 1408), Mymensingh (637), Narayanganj (644, 1324), Naogaon (1203), Noakhali (978), Norsingdi (1348, 1510), Rangamati (693), Rangpur (1293), Sirajganj (1448), Sylhet (691, 27) and Tangail (643).

Note: This species is distinguished by its brown (in old plants), rigid, julaceous habit, ovate, suddenly narrowed, short-tipped, ecostate leaves, basal angles on both sides with large triangular patches of transverse rectangular cells reaching to a considerable length on two margins.

Genus: Pterigynandrum Hedw. in Sp. Musc. : 80 (1801)

Pterigynandrum Hedw. in Lindb.: Musc. Scand.: 36 (1879) ortho. err.

Plants slender to medium sized, brownish mats. Primary stems prostrate, branches elongate, slightly julaceous, irregularly closely branched. Leaves closely imbricate, appressed when dry, slightly concave, ovate-oblong, small and narrowly acuminate, base decurrent, margin entire, nerve indistinct, sometimes distinct. Cells linear-rhomboid, shorter at apex, alar cells in oblique series, rounded-quadrate and transversely rectangular, forming a large conspicuous group extending obliquely about half way up the margins.

2. Pterigynandrum decolor (Mitt.) Broth. in Nat. Pft. 1(3): 892 (1907)

(Plate 2)

Stereodon decolor Mitt. in Musci Ind. Or.: 92 (1859)

Pylaisia brevifolia Wils. in Mitt. : id., nom. nud. in synon.

Pterigynandrum brandisii C. Muell. in Fleisch. : Hedwigia, 59: 218 (1917) nom. nud.

Leptopterigynandrum decolor (Mitt.) Fleisch. in Musci Fl. Buitenz., 4: 1496 (1923)

Creeping, branches filamentous, rigid, deep green to brownish-green, more or less glossy plants in tufts, branches terete and sometimes slightly flattened. Leaves dense, erectropatent, appressed to stem when dry, in more than one row, slightly concave, ovate, acuminate, c. $0.84 \times 0.45 \mathrm{~mm}$, margin smooth or entire. Costa usually absent, rarely double, faint, short, about $1 / 3$ to $1 / 4$ th of leaf length. Leaf cells rhomboid, non-papillose, non-porous, irregularly rhomboid at tip, c. $17.25 \times 5.25 \mu \mathrm{m}$, irregularly elongaterectangular at mid base c. $6.72 \times 8 \mu \mathrm{m}$, rhomboid at mid above c. $6.7 \times 25.5 \mu \mathrm{m}$, a few rows at basal angle are short as alar and transversely elongated c. $18.66 \times 13.23 \mu \mathrm{m}$. Main stem leaves and branch stem leaves similar, but branch leaves slightly larger in size. Sporophyte not found.

Specimen examined: Comilla (227), Moinamoti, on the bark of tree. 
Note: The species is distinguished by its creeping stem, ovate, elongated-rhomboid cells, shorter at apex, number of rows of cells at both basal angles and transversely elongated as alar.

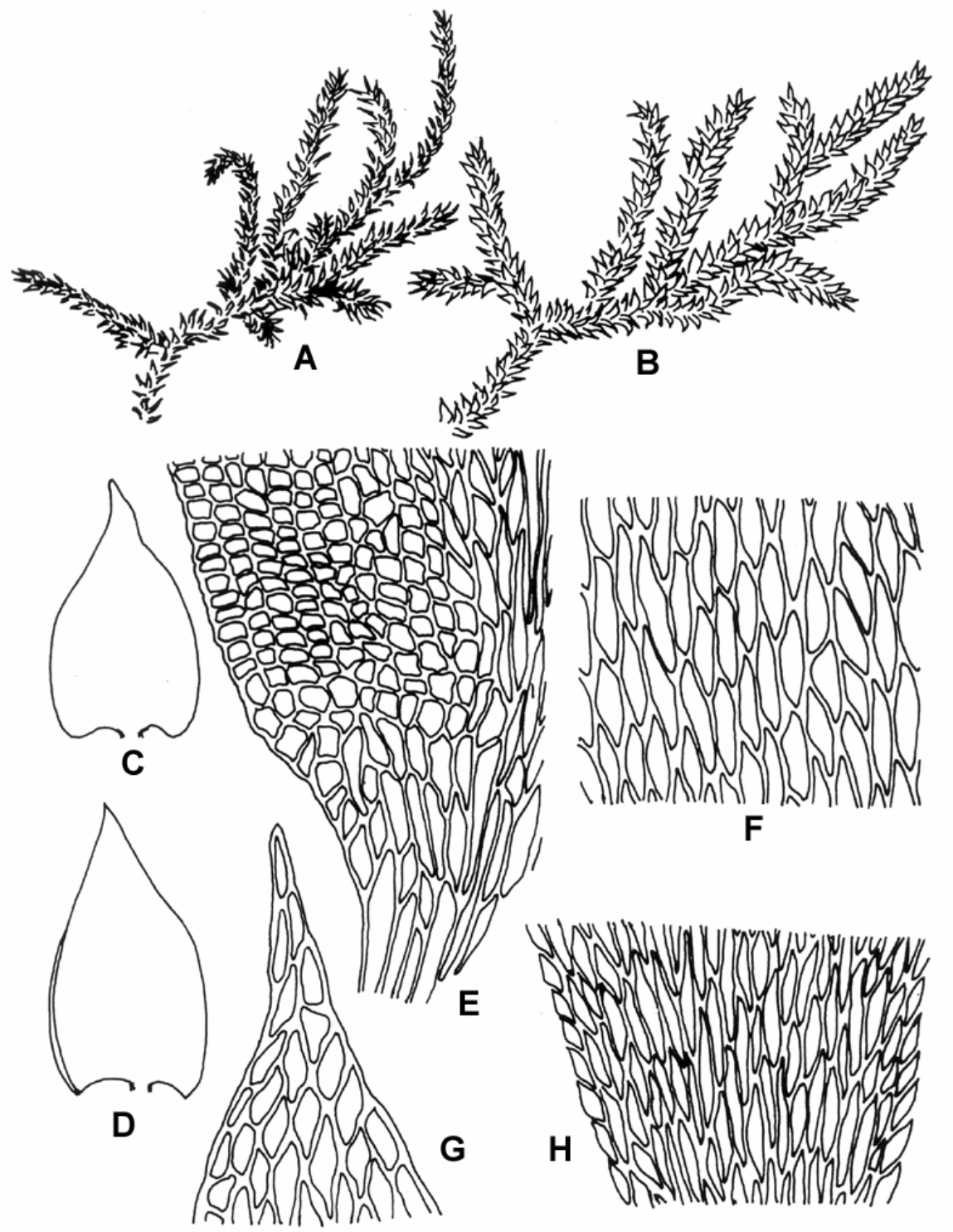

Plate 2. Pterigynandrum decolor (Mitt.) Broth. A. Dry plant $(\times 6.67)$, B. Wet plant $(\times 6.67)$, C, D. Leaves $(\times 24)$, E. Basal laminal cells $(\times 133)$, F. Upper middle laminal cells $(\times 300)$, G. Apical laminal cells $(\times 300)$, $\mathrm{H}$. Basal middle laminal cells $(\times 133)$. 
Genus: Entodon C. Muell. in Linnaea 18: 704 (1845)

Moderately robust, yellow-green mats. Main stem creeping, irregularly erect branching, leaves imbricate, ovate-lanceolate, acute, margin entire, costa short, double, cells narrow-linear, at the base large, thickened, alar cells sharply defined, quadrate, hyaline.

3. Entodon flavescens (Hook.) Jaeg. in Ber. S. Gall. Naturw. Ges. 1876-77: 293 (1878)

Neckera flavescens Hook. in Trans. Linn. Soc. Lond. 9: 314 (1808)

Stereodon schwaegricheni Mitt. in Musci Ind. Or.: 108 (1859)

Entodon schwaegrichenii (Mitt.) Broth. in Par. Index Bryol. ed. 2, 5: 151 (1906)

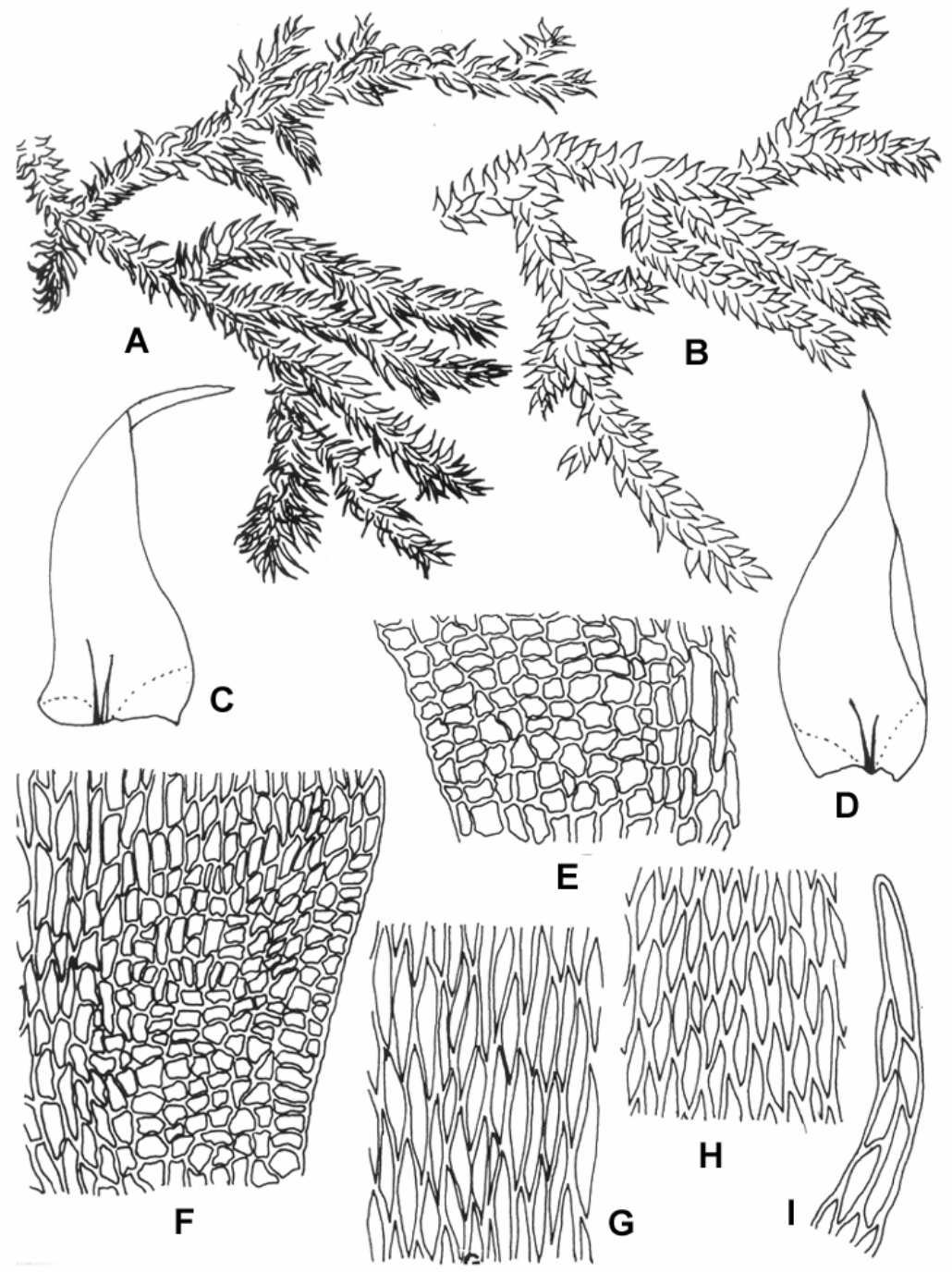

Plate 3. Entodon flavescens (Hook.) Jaeg. A. Dry plant (×6.67), B. Wet plant (×6.67), C, D. Leaves $(\times 24)$, E. Basal laminal cells on one side of midrib $(\times 200)$, F. Basal laminal cells on the other side of midrib (×200), G. Middle laminal cells (×200), H. Middle laminal cells at the upper portion of leaf $(\times 200)$, I. Apical laminal cells $(\times 200)$. 
Stem creeping, irregularly pinnately branched. Leaves complanate on main stem, triangular ovate-lanceolate c. $1.30 \times 0.48 \mathrm{~mm}$. Branch leaves erect to erectopatent, ovatelanceolate, concave, tapering at base, c.1.03 $\times 0.42 \mathrm{~mm}$, apex acute, sometimes acute acuminate, margin almost smooth. Costa two, short, unequal. Leaf cells elongated elliptic at top c. $26.4 \times 6.6 \mu \mathrm{m}$, elongate-linear to elliptic at mid lamina c. $45.9 \times 9 \mu \mathrm{m}$, alar formed of lax quadrate rectangular c. $9.79 \times 6.86 \mu \mathrm{m}$ wide cells, spreading towards costa and becoming narrower and longer above.

Specimen examined: Jessore (188), Jessore Air Force Base, on soil.

Note: This species is distinguished by its ovate-lanceolate leaves, leaf cells elongateelliptic, alar differentiated with quadrate-rectangular, lax, transparent cells.

\section{References}

Gangulee, H.C. 1980. Mosses of Eastern India and adjacent regions. A monograph. Fasc. 8. Calcutta , India. 1770-1795, 1802-1824.

Khatun, H. and Hadiuzzaman, S. 1994. Taxonomic studies of some pleurocarpic mosses of Bangladesh. Bangladesh J. Bot. 23(1): 113-122.

Khatun, H. and Hadiuzzaman, S. 1995. Addition to the pleurocarpic mosses of Bangladesh. Bangladesh J. Bot. 24(2):183-191.

Khatun, H. and Hadiuzzaman, S. 2003. Pleurocarpous mosses of Bangladesh. Family Neckeraceae-1. Bangladesh J. Plant Taxon. 10(2): 47-55.

Khatun, H. and Hadiuzzaman, S. 2004a. Pleurocarpous mosses of Bangladesh. Family Neckeraceae-2. Bangladesh J. Plant Taxon. 11(1): 43-47.

Khatun, H. and Hadiuzzaman, S. 2004b. Pleurocarpous mosses of Bangladesh. Family Erpodiaceae. Bangladesh J. Plant Taxon. 11(2): 29-32.

Khatun, H. and Hadiuzzaman, S. 2005a. Pleurocarpous mosses of Bangladesh. Family Meteoriaceae and Pterobryaceae. Bangladesh J. Plant Taxon. 12(1): 53-57.

Khatun, H. and Hadiuzzaman, S. 2005b. Pleurocarpous mosses of Bangladesh. Family Thuidiaceae and Brachytheciaceae. Bangladesh J. Plant Taxon. 12(2): 71-84.

Khatun, H. and Hadiuzzaman, S. 2006. Pleurocarpous mosses of Bangladesh. Family Symphyodontaceae and Amblystegiaceae. Bangladesh J. Plant Taxon. 13(1): 29-40.

Tixier, P. 1967. Bryophytae Indosinicae. Dacca Univ. Stud. 15(B): 1-14. 